\title{
Functional Impairment, Symptom Burden, and Clinical Outcomes Among Hospitalized Patients With Advanced Cancer
}

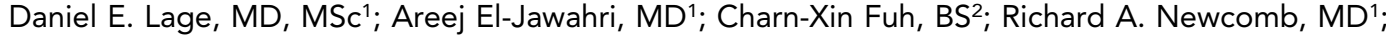 \\ Vicki A. Jackson, MD, MPH${ }^{3}$; David P. Ryan, MD¹; Joseph A. Greer, PhD; ; \\ Jennifer S. Temel, $\mathrm{MD}^{1}$; and Ryan D. Nipp, MD, MPH
}

\begin{abstract}
Background: National guidelines recommend regular measurement of functional status among patients with cancer, particularly those who are elderly or high-risk, but little is known about how functional status relates to clinical outcomes among hospitalized patients with advanced cancer. The goal of this study was to investigate how functional impairment is associated with symptom burden and healthcare utilization and clinical outcomes. Patients and Methods: We conducted a prospective observational study of patients with advanced cancer with unplanned hospitalizations at Massachusetts General Hospital from September 2014 through March 2016. Upon admission, nurses assessed patients' activities of daily living (ADLs; mobility, feeding, bathing, dressing, and grooming). Patients with any $A D L$ impairment on admission were classified as having functional impairment. We used the revised Edmonton Symptom Assessment System (ESAS-r) and Patient Health Questionnaire-4 to assess physical and psychological symptoms, respectively. Multivariable regression models were used to assess the relationships between functional impairment, hospital length of stay, and survival. Results: Among 971 patients, 390 (40.2\%) had functional impairment. Those with functional impairment were older (mean age, 67.18 vs 60.81 years; $P<.001$ ) and had a higher physical symptom burden (mean ESAS physical score, 35.29 vs $30.85 ; P<.001$ ) compared with those with no functional impairment. They were also more likely to report moderate-to-severe pain $(74.9 \%$ vs $63.1 \% ; P<.001)$ and symptoms of depression (38.3\% vs $23.6 \% ; P<.001)$ and anxiety $(35.9 \%$ vs $22.4 \%$; $P<.001)$. Functional impairment was associated with longer hospital length of stay $(\beta=1.29 ; P<.001)$ and worse survival (hazard ratio, $1.73 ; P<.001)$. Conclusions: Hospitalized patients with advanced cancer who had functional impairment experienced a significantly higher symptom burden and worse clinical outcomes compared with those without functional impairment. These findings provide evidence supporting the routine assessment of functional status on hospital admission and using this to inform discharge planning, discussions about prognosis, and the development of interventions addressing patients' symptoms and physical function.
\end{abstract}

J Natl Compr Canc Netw 2020;18(6):747-754 doi: $10.6004 /$ jnccn.2019.7385

\footnotetext{
${ }^{1}$ Department of Medicine, Division of Hematology and Oncology, Massachusetts General Hospital and Harvard Medical School, Boston, Massachusetts; ${ }^{2}$ Touro University Nevada, Henderson, Nevada; and ${ }^{3}$ Department of Medicine, Division of Palliative Care, and ${ }^{4}$ Department of Psychiatry, Massachusetts General Hospital and Harvard Medical School, Boston, Massachusetts.
}

\section{Background}

Measurement of functional status is recommended for all patients with advanced cancer, particularly older adults, ${ }^{1,2}$ but little is known about functional status among hospitalized patients with advanced cancer and its relationship to symptom burden and other clinical outcomes. Notably, hospitalized patients with cancer may actually experience more severe symptom burden and functional decline than those in the outpatient setting. ${ }^{3}$ These patients are also at risk for poor clinical outcomes, including increased risk of readmissions, frequent postacute care use, and shortened survival. ${ }^{4-7}$ In the general medicine literature, prior work has suggested that functional impairment at the time of hospital admission correlates with inferior outcomes, such as further functional decline, prolonged hospital length of stay (LoS), increased risk of hospital readmission, greater use of postacute care facilities, and worse survival. ${ }^{8-15}$ However, little is known about symptom burden and functional impairment among hospitalized patients with cancer, ${ }^{16-18}$ with even fewer data demonstrating the impact of functional impairment on subsequent clinical outcomes.

A better understanding of the relationship between the functional impairment of hospitalized patients with advanced cancer and their clinical outcomes could help inform future interventions for this population. In the general medicine population, interventions like exercise programs and discharge planning efforts have shown promise for addressing functional impairment and improving postdischarge outcomes in hospitalized patients. ${ }^{19,20}$ Despite these data, a current gap in the literature exists regarding the relationship between functional impairment, symptom burden, and clinical outcomes in hospitalized patients with cancer, thereby limiting the development of novel interventions for this population.

In the present study, we sought first to describe the rates of activities of daily living (ADLs) impairment on admission and compare characteristics of patients with

See JNCCN.org for supplemental online content. 
and without ADL impairment. We then sought to assess the relationship between patients' functional impairment at the time of hospital admission, their symptom burden, and their subsequent clinical outcomes, including LoS, rates of palliative care consultations, discharge location, readmissions, and survival. We hypothesized that patients with greater functional impairment would report a higher symptom burden and receive more inpatient palliative care, while also experiencing longer hospital LoS, greater rates of discharge to postacute care facilities, higher risk of readmissions, and inferior survival compared with those without functional impairment.

\section{Patients and Methods}

Participants

Patients were eligible for the study if they were aged $\geq 18$ years and admitted to Massachusetts General Hospital (MGH) with a diagnosis of advanced cancer. We defined patients with advanced cancer as those not being treated with curative intent, identified based on systemic therapy order entry treatment intent designation, or clinical documentation. Patients who were unable to read or respond to study questionnaires in English were excluded, as were those who were admitted for an elective or planned hospitalization, such as chemotherapy, planned surgery, or other elective procedures, or for chemotherapy desensitization. We chose to study patients with advanced cancer and unplanned hospital admissions because these patients represent a highly symptomatic population at high risk for poor clinical outcomes. ${ }^{4,7,21}$

\section{Study Procedures}

This study was approved by the Dana-Farber/Harvard Cancer Center Institutional Review Board. From September 2, 2014, to March 31, 2016, we enrolled 971 patients with advanced cancer who experienced an unplanned hospital admission at MGH. ${ }^{7}$ We identified and recruited consecutive patients with their first unplanned hospital admission during the study period by screening the daily inpatient oncology patient census. Study staff obtained written, informed consent from eligible patients on the first weekday after admission (within 2-5 days of hospitalization), and participants were given study questionnaires to complete.

\section{Sociodemographic and Clinical Measures}

We reviewed participants' electronic health records (EHRs) to collect demographic information (ie, date of birth, sex, and race) and to determine the Charlson-Deyo comorbidity score (CCS), date of diagnosis with advanced cancer, and cancer type. For multivariable models, we categorized cancer type as gastrointestinal or thoracic versus other cancer type, per our prior work. ${ }^{4}$ We conducted sensitivity analyses separating cancer type into gastrointestinal, thoracic, breast, genitourinary, or other cancer, showing no major differences in the relationship between ADL impairment and clinical outcomes.

\section{Functional Impairment Measures}

We measured functional impairment using nursing documentation of ADL impairment in the EHR. As part of standard care, nurses completed a standardized assessment of patients' ADLs within 1 day of hospital admission during the study period. Nurses assessed patients for impairment in mobility, bathing, dressing, grooming, and feeding based on a modified Katz ADL scale. ${ }^{22,23}$ Prior work has shown that these measures are comparable to validated functional measures, such as gold standard physical therapist evaluations.,11,24 We investigated rates of impairment in all 5 ADLs but dichotomized the functional impairment measure as "any ADL impairment" or "no ADL impairment" for the purposes of statistical analysis, consistent with prior work. ${ }^{11,23-25}$ ADL impairment was defined as any need for assistance by another person in completion of ADLs. As part of the standard assessment, nurses also asked patients whether they lived alone. We excluded patients with missing nursing assessment data $(n=9)$.

\section{Physical and Psychological Symptom Measures} In this study, we used the revised Edmonton Symptom Assessment System (ESAS-r) and the Patient Health Questionnaire-4 (PHQ-4) to assess physical and psychological symptoms, respectively. Both measures are widely used in the oncology setting and considered to be valid and reliable.

The self-administered ESAS-r was used to assess patients' physical symptoms, including nausea, dyspnea, lack of appetite, pain, drowsiness, well-being, and fatigue, as well as constipation because this symptom is highly prevalent in patients with advanced cancer. ${ }^{26-29}$ The questionnaire was administered at the time of consent and asked about symptoms in the past 24 hours. Patients were asked to rate each symptom on a scale of 0 to 10 , with 0 reflecting absence of the symptom and 10 reflecting the worst possible severity. We defined moderate-to-severe symptoms as ESAS-r scores of 4 to $10 .^{30}$ We computed the ESAS-Physical composite score, which included a summation of pain, fatigue, drowsiness, nausea, lack of appetite, dyspnea, and constipation (range, 0-70). ESAS-Physical scores have been used previously in the oncology setting. ${ }^{31}$

To assess patients' psychological symptoms, we used the PHQ-4, ${ }^{32,33}$ a 4 -item tool that contains two 2-item subscales assessing depression and anxiety symptoms in 
the past week. Both subscales and the composite PHQ-4 can be evaluated continuously, with subscale scores $\geq 3$ out of 6 indicating clinically significant depression or anxiety symptoms. ${ }^{32}$ We added the PHQ-4 to the study questionnaires on November 15, 2014, to include a validated measure for psychological distress $(n=783$ with completed PHQ-4).

\section{Clinical Outcomes}

We reviewed patients' EHRs to determine hospital LoS, rates of inpatient palliative care consultations, discharge location, and 90-day hospital readmissions. We categorized discharge location as home without hospice, postacute care facility (including skilled nursing facility or long-term acute care hospital), or hospice (whether provided at home, in a hospice facility, or as general inpatient care). For the discharge location analysis, we excluded patients who died during hospitalization $(\mathrm{n}=38)$.

\section{Statistical Analysis}

We compared characteristics of patients with and without ADL impairment using chi-square tests for categorical variables, 2-sample $t$ tests for continuous variables, and the Wilcoxon rank-sum tests for skewed continuous variables (LoS and months since advanced cancer diagnosis).

We used multivariable models to assess the relationship between ADL impairment and clinical outcomes, using linear regression to assess the relationship between functional impairment and ESAS-Physical symptom score, and logistic regression to assess differences in individual ESAS moderate-to-severe symptoms and differences in clinically significant depression or anxiety symptoms. Additionally, we used linear regression to assess the relationship between functional impairment and hospital LoS, and logistic regression to investigate the association between functional impairment and receipt of an inpatient palliative care consultation. Finally, we used multinomial logistic regression to analyze the relationship between functional impairment and discharge location, with a reference category of home discharge. We adjusted all regression models for age, sex, education level, CCS, whether the patient lived alone, months since advanced cancer diagnosis, and cancer type (supplemental eTable 1, available with this article at JNCCN.org). We chose to adjust for these variables a priori based on previous literature on outcomes of hospitalized patients with advanced cancer. ${ }^{4,7,24}$

We used Cox proportional hazards models to calculate hazard ratios (HRs) for censored outcomes, adjusting for age, sex, education level, CCS, months since advanced cancer diagnosis, and cancer type. To assess for risk of hospital readmission, we used time to first unplanned admission within 90 days of hospital discharge as an outcome measure, because patients who die after their index hospitalization have less time at risk for readmission, censoring patients without a readmission at 90 days and those who died within 90 days at their death date. We adjusted for index hospitalization LoS in this model given its association with readmission, in addition to the variables mentioned previously. ${ }^{34}$ We estimated survival time from the date of discharge to the date of death using the Kaplan-Meier method, and censored data from patients who were alive at the last follow-up date (September 9, 2016). In addition, we used logistic regression to evaluate the association of functional impairment with the composite dichotomous outcome, categorizing patients as dead and/or readmitted within 90 days or as alive and with no readmission within 90 days to account for early mortality. This composite outcome has been used previously in the literature for patients with a high risk of mortality. ${ }^{4,7,35-39}$

\section{Results}

Of 1,088 patients approached, $980(90.0 \%)$ agreed to participate and $971(89.2 \%)$ had data for symptoms and ADLs (supplemental eFigure 1). The sample of 971 patients had a mean age of 63.4 years (SD, 13.0; range, 20-92 years), and $49.2 \%$ were female. Participants were primarily white $(92.5 \%)$, and most had an education level above high school (60.9\%). In terms of cancer type, 31.9\% of patients $(n=310)$ had gastrointestinal cancer, $18.2 \%$ $(\mathrm{n}=177)$ had lung cancer, $10.9 \%(\mathrm{n}=106)$ had genitourinary cancer, and $7.4 \%(\mathrm{n}=72)$ had breast cancer; $31.5 \%(n=306)$ had another cancer type (Table 1). Reasons for admission included symptom management (56.0\%; $\mathrm{n}=544)$; fever or infections $(24.6 \%$; $\mathrm{n}=239)$; fatigue, weakness, or failure to thrive $(13.5 \% ; n=131)$; and metabolic disarray or laboratory abnormalities $(5.9 \%$; $\mathrm{n}=57$ ).

Overall, 390 patients $(40.2 \%)$ had at least $1 \mathrm{ADL}$ impairment, with $14.8 \%$ having 1 to 2 ADL impairments and $25.4 \%$ having $\geq 3$ (Figure 1). Among all patients, the most common functional impairments were in mobility (37.7\%), bathing (25.9\%), and dressing (24.0\%). Patients with ADL impairment were older (mean age, 67.18 vs 60.81 years; $P<.001$ ) and had higher CCS (mean, 1.13 vs $0.70 ; P<.001$ ) (Table 1).

\section{Relationship Between ADL Impairment and Symptom Burden}

Patients with at least 1 ADL impairment reported a higher physical symptom burden (ESAS-Physical; mean, 35.29 vs $30.85 ; P<.001)$. In multivariable analysis, $\mathrm{ADL}$ impairment remained significantly associated with a higher physical symptom burden $(\beta=4.80 ; 95 \% \mathrm{CI}$, 2.93-6.67; $P<.001$ ). In univariate analyses, patients with 


\section{Table 1. Patient Characteristics by ADL Impairment Status}

\begin{tabular}{|c|c|c|c|}
\hline Characteristic & $\begin{array}{c}\text { No ADL Impairment } \\
n(\%)\end{array}$ & $\begin{array}{c}\text { Any ADL Impairment } \\
n(\%)\end{array}$ & $P$ Value \\
\hline Total, n & 581 & 390 & \\
\hline Female sex & $286(49.3)$ & $192(49.2)$ & .98 \\
\hline Race/Ethnicity & & & .18 \\
\hline Hispanic & $16(2.8)$ & $6(1.5)$ & \\
\hline Asian & $14(2.4)$ & $5(1.3)$ & \\
\hline Other/Missing & $2(0.2)$ & $1(0.3)$ & \\
\hline Education & & & .06 \\
\hline CCS, mean (SD) & $0.70(1.2)$ & $1.13(1.4)$ & $<.001$ \\
\hline Cancer type & & & $<.001$ \\
\hline Gastrointestinal & $209(36.0)$ & $101(25.9)$ & \\
\hline Lung & $90(15.5)$ & $87(22.3)$ & \\
\hline Breast/Genitourinary & $81(13.9)$ & $97(24.9)$ & \\
\hline Other & $201(34.6)$ & $105(26.9)$ & \\
\hline Months since advanced cancer diagnosis, median (IQR) & $7.0(2.0-21.0)$ & $8.0(2.0-22.0)$ & .49 \\
\hline Lived alone & $94(16.2)$ & $81(20.8)$ & .07 \\
\hline
\end{tabular}

Abbreviations: ADL, activities of daily living; CCS, Charlson-Deyo comorbidity score; IQR, interquartile ratio.

ADL impairment were more likely to have moderate-tosevere constipation $(46.7 \%$ vs $36.0 \%$; $P<.001)$, pain (74.9\% vs $63.1 \%$; $P<.001)$, drowsiness $(76.6 \%$ vs $68.3 \%$; $P=.005)$, and clinically significant symptoms of depression $(38.3 \%$ vs $23.6 \% ; P<.001)$ and anxiety $(35.9 \%$ vs $22.4 \% ; P<.001$ ) (Figure 2). In multivariable analyses, impaired ADL status remained significantly associated with these individual physical and psychological symptoms.

\section{Relationship Between ADL Impairment and Clinical Outcomes}

Patients with ADL impairment had a longer median hospital LoS than patients without ADL impairment (5.0 vs 4.0 days; $P<.001$ ). In adjusted models, ADL impairment remained associated with a longer hospital LoS $(\beta=1.29 ; 95 \%$ CI, 0.66-1.93; $P<.001)$. Overall, 161 patients $(41.3 \%)$ with ADL impairment received an inpatient palliative care consultation, compared with 160 patients $(27.5 \%)$ without ADL impairment $(P<.001)$. This relationship persisted in adjusted models in which patients with ADL impairment had higher odds of receiving an inpatient palliative care consultation (odds ratio [OR], 2.41; 95\% CI, 1.79-3.24; $P<.001$ ) (Table 2).
Among patients with ADL impairment, 87 (22.3\%) were discharged to postacute care facilities compared with 31 (5.3\%) without ADL impairment. Similarly, among those with ADL impairment, 60 patients (15.4\%) were discharged to hospice compared with $28(4.8 \%)$ without ADL impairment. In a multinomial logistic regression model for discharge location, with discharge home as the reference category, patients with ADL impairment had greater odds of discharge to a postacute care facility (OR, $5.41 ; 95 \% \mathrm{CI}, 3.41-8.58 ; P<.001)$ or hospice (OR, 5.58; 95\% CI, 3.40-9.16; $P<.001)$ (Table 2).

Overall, $43.4 \%$ of patients were readmitted within 90 days. According to a multivariable Cox regression analysis of readmissions within 90 days, ADL impairment was not significantly associated with readmissions (HR, 1.12; 95\% CI, 0.91-1.38; $P=.303$ ). To help account for early mortality, we calculated odds of death or readmission within 90 days and, in this model, patients with any ADL impairment had significantly higher odds of death or readmission within 90 days (OR, 2.25; 95\% CI, 1.67-3.03; $P<.001)$. Patients with any ADL impairment had a median survival after discharge of 78.5 days compared with 163.0 days for those without ADL impairment (log-rank $P<.001)$. According to a multivariable Cox regression 


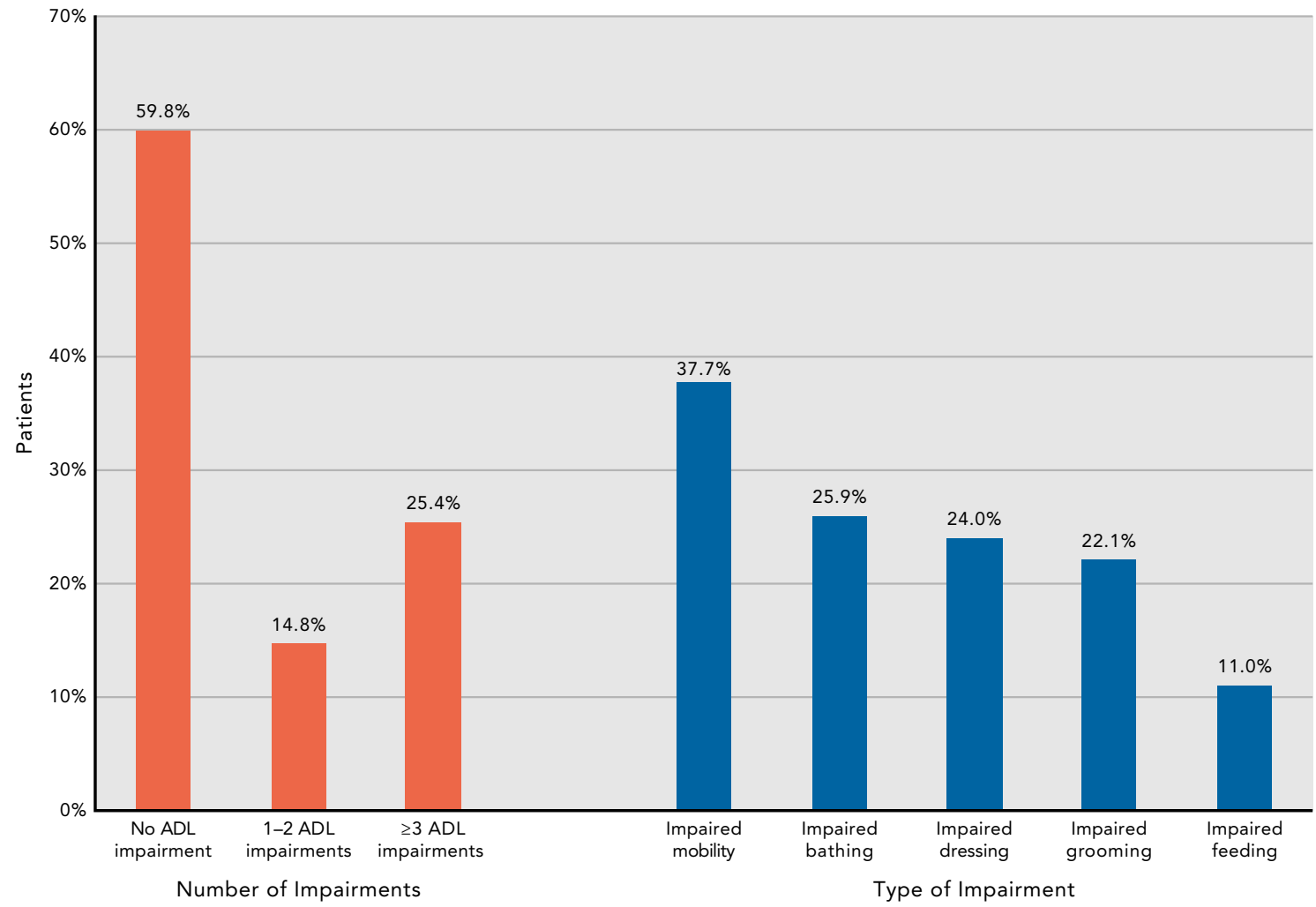

Figure 1. Impairments among all patients $(n=971)$. Abbreviation: $A D L$, activities of daily living.

analysis, ADL impairment was associated with a significantly greater hazard for death (HR, 1.73; 95\% CI, $1.47-2.04 ; P<.001$ ) (Figure 3 ).

\section{Discussion}

We found that a substantial proportion of hospitalized patients with advanced cancer present with functional impairment, and these patients represent a population at particularly high risk for poor clinical outcomes. Specifically, we discovered relationships between functional impairment and increased physical and psychological symptom burden. We also showed that functional impairment has important associations with longer hospital LoS, discharge to a location other than home, and inferior survival. Collectively, these findings underscore the importance of integrating functional assessment into the care of hospitalized patients with advanced cancer, given that nearly half of these patients had functional impairment in our study. This information can help guide discharge planning, discussions about prognosis, and targeting of limited palliative care resources to patients at risk for poor clinical outcomes.

Assessment of patients' functional impairment may not be routinely integrated into physicians' decisionmaking on admission, despite the recommendations of several professional societies. ${ }^{2,10}$ We found that $>40 \%$ of patients screened positive for functional impairment in our study, which is consistent with prior work and underscores the need for routine functional assessments to identify those individuals in need of additional services. ${ }^{17}$ Our results suggest that data about functional impairment derived from routine nursing assessments can identify patients with high symptom burden, longer hospital LoS, increased risk of discharge to a postacute care facility, and worse survival. Furthermore, identifying a high-risk population of hospitalized patients with cancer could help with targeting interventions for these patients. For example, models of care in the general medicine literature could be adapted for an inpatient oncology population, including home-based postdischarge services and care transition interventions. ${ }^{19,20,40}$ Other promising care models to consider for functionally impaired hospitalized patients with cancer include inhospital exercise programs, ${ }^{41}$ telerehabilitation, ${ }^{42}$ and alternatives to hospitalizations, such as hospital-athome interventions. ${ }^{43}$ Thus, by simply capturing basic information about patients' ADLs at the time of hospital admission, clinicians could use this information to guide discussions with their patients about the need to better manage symptoms, address functional impairment through 


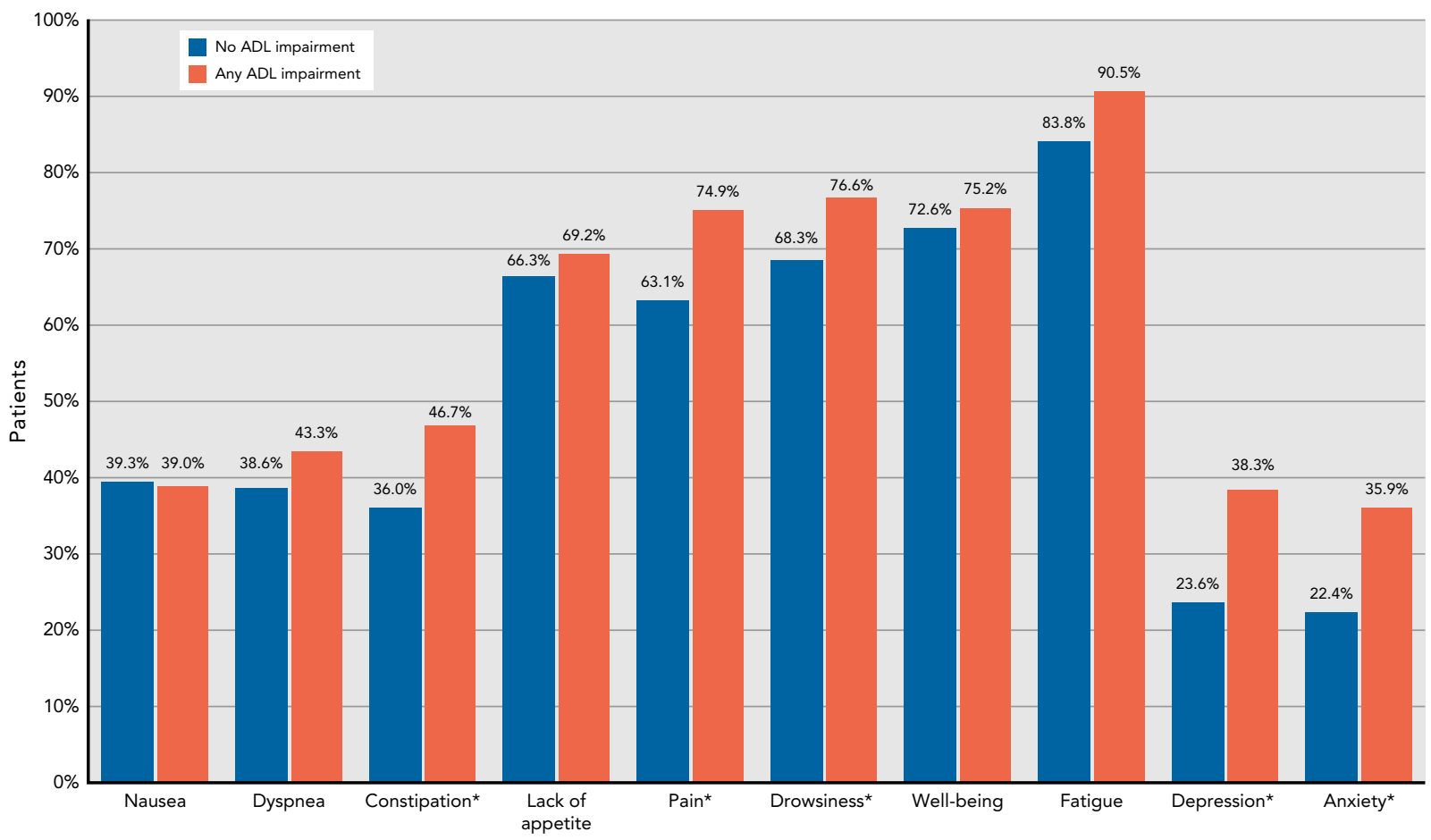

Figure 2. Physical and psychological symptom burden by ADL impairment status.

Abbreviation: ADL, activities of daily living.

${ }^{\star} P<.01$.

novel interventions, and plan for posthospital transitions of care.

Our finding of a relationship between functional impairment and greater physical and psychological symptom burden during hospitalization has several implications for the integration of palliative care and symptom monitoring interventions into inpatient oncology care. Functionally impaired patients in our study received higher rates of inpatient palliative care consultation, suggesting that clinical teams often recognize that this population may have unmet supportive care needs. However, even among this symptomatic and functionally impaired population, fewer than half received a palliative care consultation in our study. Efforts to integrate palliative care earlier and more frequently could help address these patients' symptom burden and may also enhance their ability to develop attainable functional goals, improve prognostic awareness, and formulate a plan to adequately support transitions out of the hospital. ${ }^{4,44-46}$ Moreover, we found a novel association

Table 2. Multivariable Models for Association Between any ADL Impairment and Clinical Outcomes

\begin{tabular}{|lccc|}
\hline Clinical Outcome & $\begin{array}{c}\text { No ADL Impairment } \\
\mathbf{n}(\%)\end{array}$ & $\begin{array}{c}\text { Any ADL Impairment } \\
\mathbf{n}(\%)\end{array}$ & $\begin{array}{c}\text { Adjusted Coefficient for ADL } \\
\text { Impairment } \\
\text { OR (95\% Cl) }\end{array}$ \\
\hline Total, $\mathrm{n}$ & 581 & 390 & $\beta=1.29(0.66-1.93)^{\star}$ \\
\hline Length of stay, median (IOR), $d$ & $4.0(3.0-7.0)$ & $5.0(4.0-9.0)$ & $2.41(1.79-3.24)^{\star}$ \\
\hline Inpatient palliative care consultation & $160(27.5)$ & $161(41.3)$ & $5.41(3.41-8.58)^{\star}$ \\
\hline Discharge to postacute care vs home & $31(5.3)$ & $87(22.3)$ & $5.58(3.40-9.16)^{\star}$ \\
\hline Discharge to hospice vs home & & $60(15.4)$ & $2.25(1.67-3.03)^{\star}$ \\
\hline Death or readmission in 90 days & $28(4.8)$ & $294(75.4)$ & 2.4 \\
\hline
\end{tabular}

All models adjusted for age, sex, education, Charlson-Deyo comorbidity score, whether patient lived alone, months since advanced cancer diagnosis, and cancer type (gastrointestinal/thoracic vs other cancer).

Abbreviations: ADL, activities of daily living; IQR, interquartile ratio; $O R$, odds ratio.

apatients who died during hospitalization $(n=38)$ were excluded from multivariable analysis of discharge location.

$\star P<.001$. 


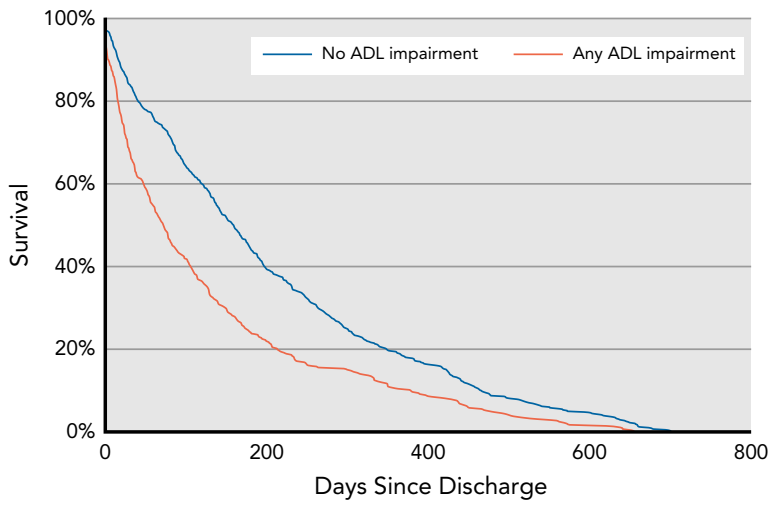

Figure 3. Survival by ADL impairment status.

Abbreviation: $A D L$, activities of daily living.

between functionally impaired individuals and increased psychological symptoms, which merits additional study to understand the underlying mechanism and supports the need to integrate psychosocial support services into these patients' care. Finally, with the increasing data demonstrating the benefits of symptom monitoring interventions, ${ }^{47-49}$ efforts to understand how these types of interventions could help address hospitalized patients' functional outcomes are needed. Thus, future efforts to enhance care quality and outcomes for hospitalized patients with cancer should involve developing and testing interventions that integrate routine monitoring of both functional impairment and symptom burden for these patients.

This work has several limitations. First, we conducted this study at a single hospital using a relatively homogenous patient sample with respect to sociodemographic characteristics, which may limit the generalizability of our results. Second, we examined impairment in ADLs as assessed by nurses at the bedside, and although this approach has been validated and published previously, ${ }^{4,11,24}$ we lacked information about physical therapy consultation rates and the results of these evaluations, as well as information on prehospital functional status measures. An important future question is whether these impairments can be addressed by physical therapy or other interventions. We also lacked data on traditional performance status measures, such as Karnofsky and ECOG performance status. Furthermore, we could not assess longitudinal trajectories of ADL impairment and symptom burden, because we focused on data at a single time point at the time of hospital admission. We also could not assess readmissions to hospitals outside our system, which could lead to underreporting of readmissions. In addition, we lacked data regarding hospice enrollment that may have occurred days or weeks after hospital discharge, as well as hospice LoS and site of death, which represent important end-of-life outcomes in this population.

\section{Conclusions}

A substantial proportion of hospitalized patients with advanced cancer experience functional impairment, and we found that functional impairment in this population is associated with a higher symptom burden, increased healthcare utilization, and inferior survival. These findings provide data supporting routine assessment of patients' functional outcomes during hospital admission and the use of this information to inform discharge planning, target palliative care and psychosocial support services, and guide prognostic discussions between patients and their care team in order to enhance quality of life and clinical outcomes for these patients.

Submitted September 1, 2019; accepted for publication December 4, 2019

Author contributions: Study concept and design, acquisition of data, or analysis and interpretation of data: All authors. Manuscript preparation and critical revision: All authors.

Disclosures: Dr. Ryan has disclosed that he is a consultant for MPM Capital. Dr. Greer has disclosed that he recieves grant/research support from and is a consultant for Gaido Health/BCG Digital Ventures. The remaining authors have disclosed that they have not received any financial consideration from any person or organization to support the preparation, analysis, results, or discussion of this article.

Funding: This work was supported by funding from Massachusetts General Hospital (MGH Cancer Center Funds).

Correspondence: Daniel E. Lage, MD, MSc, Department of Medicine, Division of Hematology and Oncology, Massachusetts General Hospital Cancer Center, 55 Fruit Street, Yawkey Building, Boston, MA 02114. Email: dlage@mgh.harvard.edu

\section{References}

1. Dotan E, Walter LC, Baumgartner J, et al. NCCN Clinical Practice Guidelines in Oncology: Older Adult Oncology. Version 1.2019. Accessed August 24, 2019. To view the most recent version, visit NCCN.org

2. Mohile SG, Dale W, Somerfield MR, et al. Practical assessment and management of vulnerabilities in older patients receiving chemotherapy: ASCO guideline for geriatric oncology. J Clin Oncol 2018;36: 2326-2347.

3. Portenoy RK, Thaler HT, Kornblith AB, et al. The Memorial Symptom Assessment Scale: an instrument for the evaluation of symptom prevalence, characteristics and distress. Eur J Cancer 1994;30:1326-1336.

4. Lage DE, Nipp RD, Arpino S, et al. Predictors of posthospital transitions of care in patients with advanced cancer. J Clin Oncol 2018; 36:76-82.

5. Brooks GA, Li L, Uno H, et al. Acute hospital care is the chief driver of regional spending variation in Medicare patients with advanced cancer Health Aff (Millwood) 2014;33:1793-1800.

6. Brooks GA, Li L, Sharma DB, et al. Regional variation in spending and survival for older adults with advanced cancer. J Natl Cancer Inst 2013; 105:634-642.

7. Nipp RD, El-Jawahri A, Moran SM, et al. The relationship between physical and psychological symptoms and health care utilization in hospitalized patients with advanced cancer. Cancer 2017;123:4720-4727.

8. Greysen SR, Stijacic Cenzer I, Boscardin WJ, et al. Functional impairment: an unmeasured marker of Medicare costs for postacute care of older adults. J Am Geriatr Soc 2017;65:1996-2002. 
9. Barnett ML, Grabowski DC, Mehrotra A. Home-to-home time-measuring what matters to patients and payers [abstract]. New Engl J Med 2017;377: 4-6.

10. Wald HL, Ramaswamy R, Perskin $\mathrm{MH}$, et al. The case for mobility assessment in hospitalized older adults: American Geriatrics Society white paper executive summary. J Am Geriatr Soc 2019;67:11-16.

11. Pavon JM, Sloane R, Morey MC, et al. Inpatient mobility measures as useful predictors of discharge destination in hospitalized older adults. J Am Geriatr Soc 2017;65:224-226.

12. Brown CJ, Foley KT, Lowman JD Jr, et al. Comparison of posthospitalization function and community mobility in hospital mobility program and usual care patients: a randomized clinical trial. JAMA Intern Med 2016;176:921-927.

13. Buurman BM, Han L, Murphy TE, et al. Trajectories of disability among older persons before and after a hospitalization leading to a skilled nursing facility admission. J Am Med Dir Assoc 2016;17:225-231.

14. Jette DU, Stilphen M, Ranganathan VK, et al. AM-PAC "6-Clicks" functional assessment scores predict acute care hospital discharge destination. Phys Ther 2014;94:1252-1261.

15. Brown CJ, Friedkin RJ, Inouye SK. Prevalence and outcomes of low mobility in hospitalized older patients. J Am Geriatr Soc 2004;52: 1263-1270.

16. Repetto L, Fratino L, Audisio RA, et al. Comprehensive geriatric assessment adds information to Eastern Cooperative Oncology Group performance status in elderly cancer patients: an Italian Group for Geriatric Oncology Study. J Clin Oncol 2002;20:494-502.

17. McCarthy EP, Phillips RS, Zhong Z, et al. Dying with cancer: patients' function, symptoms, and care preferences as death approaches. J Am Geriatr Soc 2000;48(Suppl 1):S110-121.

18. Flood KL, Carroll MB, Le CV, et al. Geriatric syndromes in elderly patients admitted to an oncology-acute care for elders unit. J Clin Oncol 2006;24: 2298-2303.

19. Coleman EA, Parry C, Chalmers $\mathrm{S}$, et al. The care transitions intervention: results of a randomized controlled trial. Arch Intern Med 2006;166: 1822-1828.

20. Naylor MD, Brooten D, Campbell R, et al. Comprehensive discharge planning and home follow-up of hospitalized elders: a randomized clinical trial. JAMA 1999;281:613-620.

21. Rocque GB, Barnett AE, Illig LC, et al. Inpatient hospitalization of oncology patients: are we missing an opportunity for end-of-life care? J Oncol Pract 2013;9:51-54.

22. Katz S, Ford AB, Moskowitz RW, et al. Studies of illness in the aged. The index of ADL: a standardized measure of biological and psychosocial function. JAMA 1963;185:914-919.

23. Walter LC, Brand RJ, Counsell SR, et al. Development and validation of a prognostic index for 1-year mortality in older adults after hospitalization. JAMA 2001;285:2987-2994.

24. Lage DE, Jernigan MC, Chang $Y$, et al. Living alone and discharge to skilled nursing facility care after hospitalization in older adults. J Am Geriatr Soc 2018;66:100-105.

25. Lee SJ, Lindquist K, Segal MR, et al. Development and validation of a prognostic index for 4-year mortality in older adults. JAMA 2006;295: 801-808.

26. Watanabe SM, Nekolaichuk C, Beaumont C et al. A multicenter study comparing two numerical versions of the Edmonton Symptom Assessment System in palliative care patients. J Pain Symptom Manag 2011;41: 456-468.

27. Bruera E, Kuehn N, Miller MJ, et al. The Edmonton Symptom Assessment System (ESAS): a simple method for the assessment of palliative care patients. J Palliat Care 1991;7:6-9.

28. Rhondali W, Nguyen L, Palmer L, et al. Self-reported constipation in patients with advanced cancer: a preliminary report. J Pain Symptom Manag 2013;45:23-32.
29. Richardson LA, Jones GW. A review of the reliability and validity of the Edmonton Symptom Assessment System. Curr Oncol 2009;16:55

30. Selby D, Cascella A, Gardiner K, et al. A single set of numerical cutpoints to define moderate and severe symptoms for the Edmonton Symptom Assessment System. J Pain Symptom Manag 2010;39:241-249.

31. Hui $D$, Shamieh $O$, Paiva $C E$, et al. Minimal clinically important difference in the physical, emotional, and total symptom distress scores of the Edmonton Symptom Assessment System. J Pain Symptom Manage 2016; 51:262-269.

32. Kroenke K, Spitzer RL, Williams JB, et al. An ultra-brief screening scale for anxiety and depression: the PHQ-4. Psychosomatics 2009;50:613-621.

33. Löwe $B$, Wahl I, Rose M, et al. A 4-item measure of depression and anxiety: validation and standardization of the Patient Health Questionnaire-4 (PHQ-4) in the general population. J Affect Disorders 2010;122:86-95.

34. Donzé JD, Williams MV, Robinson EJ, et al. International validity of the HOSPITAL score to predict 30-day potentially avoidable hospital readmissions. JAMA Intern Med 2016;176:496-502.

35. El-Jawahri A, Chen YB, Brazauskas R, et al. Impact of pre-transplant depression on outcomes of allogeneic and autologous hematopoietic stem cell transplantation. Cancer 2017;123:1828-1838.

36. Brown JR, Conley SM, Niles NW II. Predicting readmission or death after acute ST-elevation myocardial infarction. Clin Cardiol 2013;36:570-575.

37. Ballen KK, Joffe S, Brazauskas R, et al. Hospital length of stay in the first 100 days after allogeneic hematopoietic cell transplantation for acute leukemia in remission: comparison among alternative graft sources. Biol Blood Marrow Transplant 2014;20:1819-1827.

38. Brown PM, Ezekowitz JA. Composite end points in clinical trials of heart failure therapy: how do we measure the effect size? Circ Heart Fail 2017; 10:e003222.

39. Glance LG, Li Y, Dick AW. Impact on hospital ranking of basing readmission measures on a composite endpoint of death or readmission versus readmissions alone. BMC Health Serv Res 2017;17:327.

40. Naylor MD, Shaid EC, Carpenter D, et al. Components of comprehensive and effective transitional care. J Am Geriatr Soc 2017;65:1119-1125

41. Martínez-Velilla N, Casas-Herrero A, Zambom-Ferraresi F, et al. Effect of exercise intervention on functional decline in very elderly patients during acute hospitalization. JAMA Intern Med 2019;179:28-36.

42. Cheville AL, Moynihan T, Herrin J, et al. Effect of collaborative telerehabilitation on functional impairment and pain among patients with advanced-stage cancer: a randomized clinical trial. JAMA Oncol 2019;5: 644-652.

43. Federman AD, Soones $T$, DeCherrie LV et al. Association of a bundled hospital-at-home and 30-day postacute transitional care program with clinical outcomes and patient experiences. Jama Intern Med 2018;178: 1033-1040.

44. Coleman EA, Min S, Chomiak A, et al. Posthospital care transitions: patterns, complications, and risk identification. Health Serv Res 2004;39: 1449-1465.

45. Ackerly CD, Grabowski DC. Post-acute care reform-beyond the ACA N Engl J Med 2014;370:689-691.

46. Flint LA, David DJ, Smith AK. Rehabbed to death. N Engl J Med 2019;380 408-409.

47. Basch E, Deal AM, Kris MG, et al. Symptom monitoring with patientreported outcomes during routine cancer treatment: a randomized controlled trial. J Clin Oncol 2016;34:557-565.

48. Strasser F, Blum D, von Moos R, et al. The effect of real-time electronic monitoring of patient-reported symptoms and clinical syndromes in outpatient workflow of medical oncologists: E-MOSAIC, a multicenter cluster-randomized phase III study (SAKK 95/06). Ann Oncol 2016;27: 324-332.

49. Nipp R, El-Jawahri A, Ruddy M, et al. Pilot randomized trial of an electronic symptom monitoring intervention for hospitalized patients with cancer. Ann Oncol 2019;30:274-280.

\section{See JNCCN.org for supplemental online content.}


Supplemental online content for:

\section{Functional Impairment, Symptom Burden, and Clinical Outcomes Among Hospitalized Patients With Advanced Cancer}

Daniel E. Lage, MD, MSc; Areej El-Jawahri, MD; Charn-Xin Fuh, BS; Richard A. Newcomb, MD;

Vicki A. Jackson, MD, MPH; David P. Ryan, MD; Joseph A. Greer, PhD;

Jennifer S. Temel, MD; and Ryan D. Nipp, MD, MPH

J Natl Compr Canc Netw 2020;18(6):747-754

eFigure 1: Cohort Selection

eTable 1: Multivariable Models Examining the Association Between ADL Impairment and Clinical Outcomes 


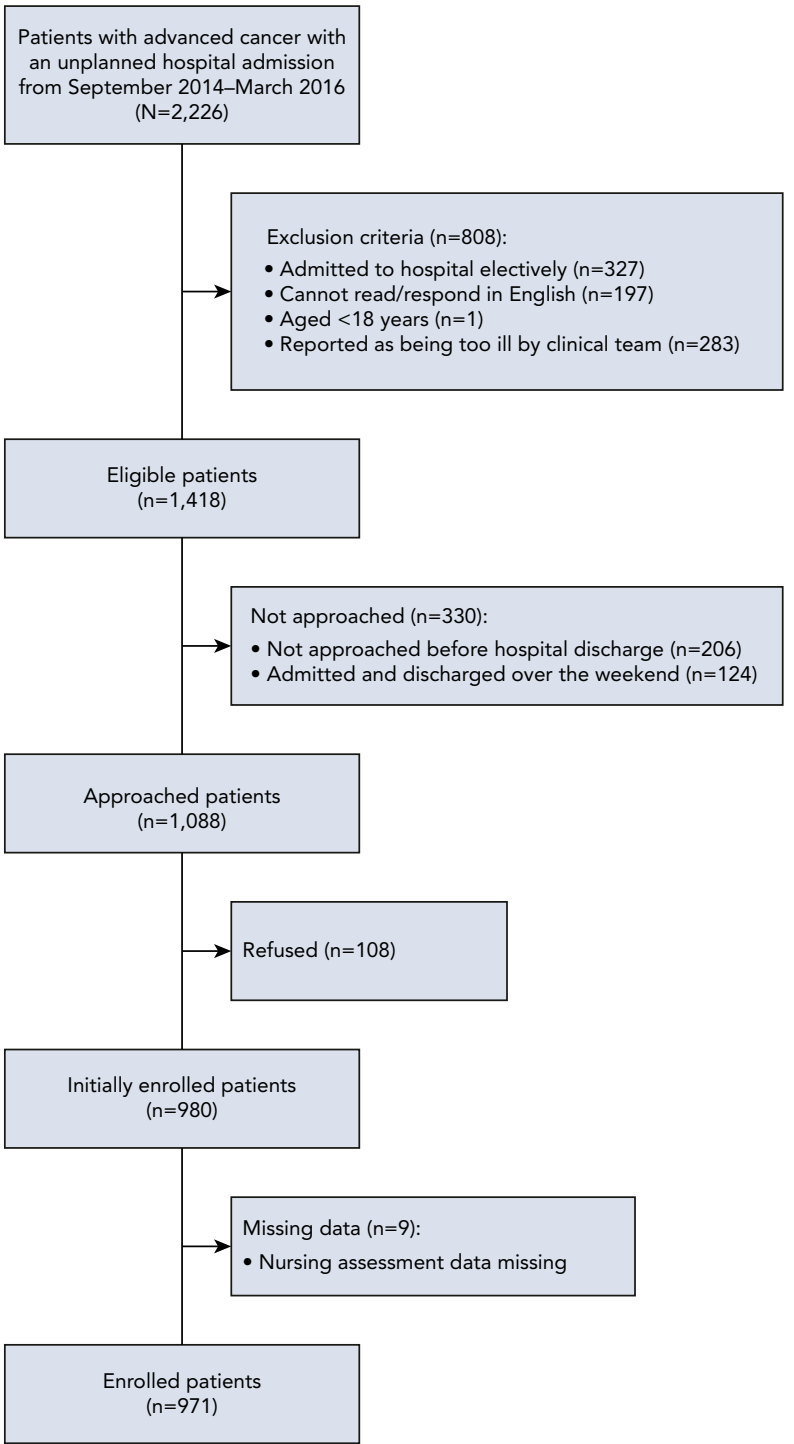

eFigure 1. Cohort selection. 


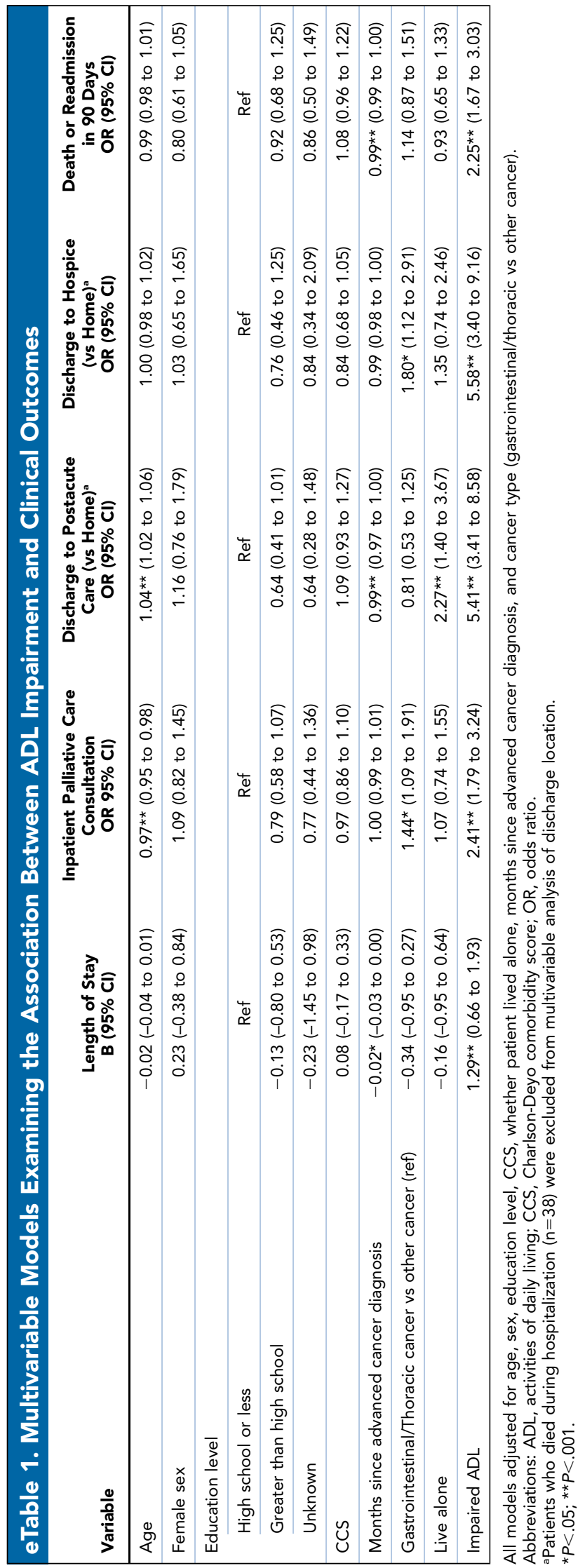

